\title{
Palm print Recognition using 2D Fourier Transformation and Integration Function
}

\author{
Abhilove Kumar, Apoorv Mishra
}

Department of Computer Science and Engineering, Naraina Vidya Peeth Engineering \& Management Institute, Kanpur, India

\begin{abstract}
Article Info

Volume 7, Issue 3

Page Number: 555-560

Publication Issue :

May-June-2021

Article History

Accepted : 18 June 2021

Published : 25 June 2021

Palm print authentication technique is very powerful technique as compare with other technique and it is also very friendly with the user and environment. Palm is the mainly inner part of the hand which shows different features as compare with other in this it technique it mainly provide the path to authenticate the user or modify the user. In this technique we use phase based matching algorithm and if in this problems will occur then it reduce by using of Fourier Transform technique and Integration system. These techniques extract the features of the palm to modify it and there are so many features for extraction. In this we authenticate the user with applying some of the transformation where when we extract the feature vector then it will not give the accurate result. That's why we using this transformation technique in this which is very useful for the user.
\end{abstract}

Keywords : Machine Learning, Palm print, Histogram, Minutiae

\section{INTRODUCTION}

User identification process is highly recommendable for human being because it is basically based upon the physiological attributes and Behavioral attributes. Biometric technologies are becoming day-by-day a popular authentication process only because of its verification process. With an augment in level of breaking safety precautions and transaction-frauds, there is need for highly locked identification and personal verification technologies are becoming clear. In today's era there are so many hackers are there which detect the system with help of its hacking so to prevent it we use the highly probable authentic technology and it is only the palm print biometric feature.

As we see day by day the hacking attacks are increasing and it may hack our system or hack our data. And in today's generation as we see different hackers use that type of algorithms which we cannot imagine ever and stole our data with use of algorithm as they break the passwords of user in fact in this so many safety procedure they follow. Then to prevent from these hacking we use the proper authentication process and the palm print biometric is the best method to resolve out from all problems. 
This application is very useful in account purposes because it mainly generates the passwords of that account and it is different from others and that is the only reason it is safe. In case of passwords remembering commonly we forget the passwords and then data is loosed so if we using the palm print biometrics in financial purposes we never faced that type of problem and this generate our user Id and we did not need to remember that passwords. When it creates the user Id it provides the uniqueness to the particular human being and linked it with our system.

\section{Related Work}

In this we study about the earlier work done on palm print technology and there are so many researchers who give the best result on this technique of biometric authentication. The concept of biometric [1] authentication system is so old because it is continuously increases day by day and it is generally highly vulnerable to optimized the data and it is mainly considered as the optimization era.

In 1858, Sir w. Herchel, in Civil service of India [2] who took this palm authentication technique and take it for long once a issue not come. After few years, in 1870 a new method came on palm print technique and it is work according to the human body move which given by Sir E. Henry.

In 2002, W. Kong and D. Zhang [3] has used many of other techniques in which they used the Gabor Filters for palm print technique and it is evolved with hamming distance concept. They take its resolution as image per image and fixed its rate according with the palm print path. He takes so many resolutions which gives it an accurate result and it is simultaneously involved the path of this technique.

After one year in 2003, D. Zhang et al. [4] again applied the filters in low resolution and its template is mainly gives the accurate result before than good in that case it is originally confirmed that path which is highly accumable path of that data and in using with of various techniques involved into it.

Again in2004, L. Zhang and D. Zhang [5] occupied another parameter in palm print where they extract its features which is corresponding with each other and here they use the wavelet transform series here they identify the human being's palm and they gives the accurate result which is approx at $98 \%$ accurate.

In this they use the separability feature which acquired the partition features which is generally modified the path [6]. There are few others image processing techniques were used earlier in this field [7-9].

\section{Proposed Methodology}

In this section an algorithm has been discussed which phase base is matching for palm detection and localization which is based on palm edges and it has been proposed. The technique is based on the palm feature that is, "creases, ridges and wrinkles". The objective of algorithm is to detect the palm to consider the muscles; it has only three areas to cover which are lower palm, middle palm, upper palm, palm lines etc. In figure 1 shows the area where these terms are considered. The algorithm for palm detection is constrained to figures in which only one hand palm is here but a slight alteration can be applied to algorithm for detecting multiple palms present in any of the figure. With the help of 2D integration technique we conclude the figure of palm of different human being is detected in a very fast speed and takes lesser amount of time to detect a criminal or detect a human being because it is that type of technique which is used in so many places to detect a person. 


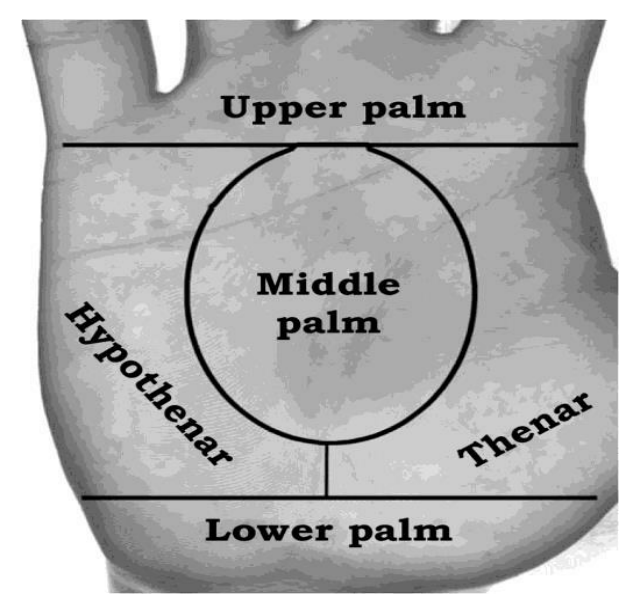

Figure 1. Palm Areas

In this approach we detect the skin complexion basically it is not the main factor because all the human being have almost same palm complexion but the changes occur in its only palm line and little bit in palm complexion. In this the complexion of color is initiate its red, green and blue light which is added together in various ways to give the extensive display of colors. With the help of three components that $\mathrm{R}$, $G$ and $B$, it gives the luminance information and it also have the good co-relation with each other.

Here, we discuss the given four steps on the diagram which intended according to the given approaches as we seen in Figure 2. Now we see in the first stanza as it stacked the palm images outlines when we click the clicks and upload onto the database. In the second stanza, it evaluate the face with the used of algorithm and it appears to be clear because when we upload the images from the database it constituently identify the images. Here is the third stanza, which modifies the comparisons with minutiae terms of palm like ridges, wrinkles and etc. and at the last stanza it detects the complexion of skin which identified by transformation of the components and it is matched the clicks from the database. Linear matching technique is further categorized by the all stanza which considered the path and matched the data from stored document.

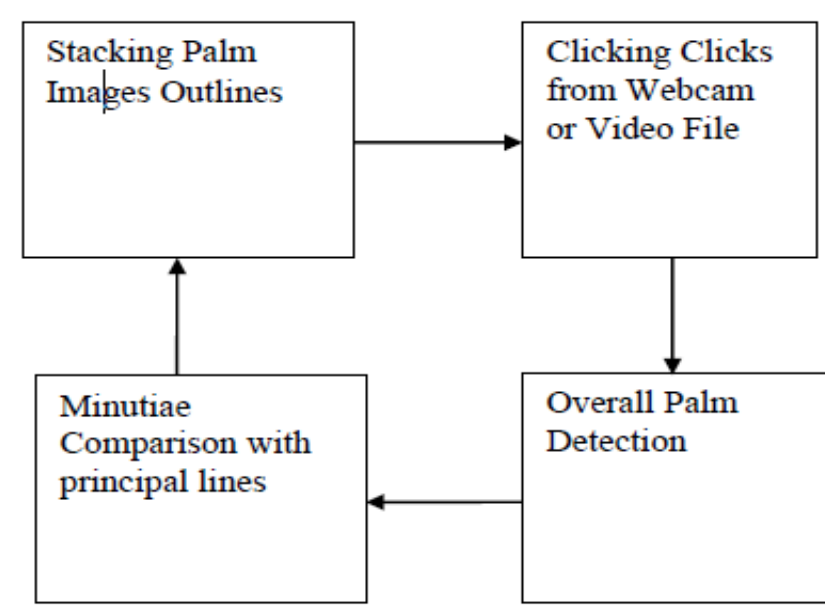

Figure 2. Palm print Identification Stages

When we stacked the palm images then here it is basically related with its complexion and its size also because it is basically depend on these factors. As we captured the image and matched image is not properly set onto the path then it is react with rejected image. In this stage firstly we upload all the clicks or the images as we seen figure 2 Grasp, pointer, Minutiae and at last on palm where it checks all the circumstances of palm prints which we used in the path of clarity. It is fixed the path on the stanza which combined all the stages and with help of these stages.

After verifying the stanza we processed on the second one and it is variable to all of other stanza and for all these firstly we have to check the first one because it is the basic and it is directly converted the image into gray level format. In this we used to different pixels to upload the images as $(50 \times 50$ pixels $)$ to demonstrate the images and the features exerts here images are clicked with the camera which has the higher frequencies to develop the feature with the different database images.

\section{Experimental work}

In the experiment an estimation of effects of changes in palm image due to change in illumination conditions and viewpoint is done. Various functions 
are applied on palm images which recover it by different situations. Different representations formed after applying various filters were taken. One representation of a person's palm image was taken and was matched against same representation of different person's images and image of same person taken in different viewpoint and illumination condition. All the functions and representations that were used are explained in next section.

As the task was to check the possibility of problem of palm print detection while there are changes in illumination direction and viewpoint, we have taken a database of 125 different images which belonged to 25 different persons with 5 palm images per person. Lighting and viewing directions were strictly controlled to ensure uniformity in the pictures taken and it taken is very accurately. All the images were of palm and no occlusion due to rings on fingers or any other factor was there.

Different conditions for the images are described as follows.

(A) In the first image light was coming from left direction and side image of palm was taken.

(B) The light source and camera position continued same with respect to each other but the human being's palm was rotated by $15^{\circ}$ toward left direction as compared to first case.

(C) The light source and camera position continued same with respect to each other but the human being's palm was rotated by $34^{\circ}$ toward left direction as compared to first case.

(D) The viewpoint of the camera continued same but the direction of light source was changed from left direction to right direction.

(E) The viewpoint of camera continued same but a different light source from front direction was used.

The images were further extracted using a mask. In first the palm images were extracted manually and it is simulate not to be any of one other images. The average height to width ratio of palm region was calculated and was found to be 1.52 . Finally the mask was generated which was an ellipse with eccentricity equal to 1.52 which had center at center of palm region. Various images are taken of same human being to detect it with the some algorithms.

\subsection{Distance Measurements}

For matching, point wise distance appraise was taken. Point wise distance between different images with different illustrations was calculated. Point wise distance is used in for distance measurement which can be described as follows.

It is average distance between gray level values of all pairs of corresponding pixels of two images to be matched accurately with compared to any of one other feature.

$$
\text { Distance }_{\left(\text {Image }_{1}, \operatorname{Image}_{2}\right)}=\frac{1}{n}\left|\operatorname{Imagel}(x)-\operatorname{Image}_{2}(x)\right|
$$

here $\mathrm{n}$ is number of pixels common in masks of Image1 and Image2, Image1 $(x)$ and Image2 $(x)$ are gray level values of corresponding pixels of two images to be matched.

\section{Result and Discussion}

The test was executed on 130 different palm images of 26 persons with 5 images per person described in earlier section. The evaluation was done based on hit percent of a particular filter. For each palm image distance between a human being's palm print image with change in illumination or viewpoint and the first image (one with left illumination without any rotation) of the same person were taken. If this distance was less than all other images of different persons belonging to the same class (same class here means the images of all the persons which had same illumination and viewing directions) the 
identification was considered as a hit. The percent of hit for all the last four classes i.e. with $15^{\circ}$ rotation, $34^{\circ}$ rotation, same light source from right direction, a different front light source with some ambiance were calculated and it is done accurately with the help of some beneficial techniques. One can easily deduce that larger changes in viewing directions are not acceptable in case of palm biometrics. A good hitpercent is achieved for the images in which viewing direction changed by $15^{\circ}$.
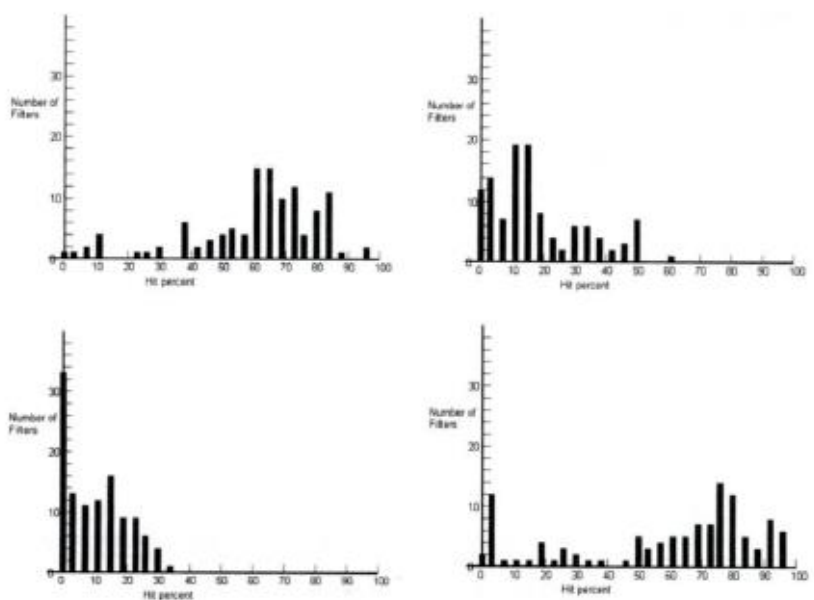

Figure 3. Histograms of hit percent for 113 various filters for different kind of variations (a) $15^{\circ}$ rotation (b) ( a) $34^{\circ}$ rotation (c) same light source from right direction (d) a different light source with some ambiance were calculated.

Hit percent for filters is shown in table above. From the table it is assumed that simple smoothing filters give good results for nearly similar images but are not very robust to changes as it can be seen that they are very much affected by changes in light direction. Also these illustrations give very poor result in case of changes in viewpoint. Very good result in case of mean function on $15^{\circ}$ rotation can be clarified by small change in gray level values due to small change in viewing direction.
Table 1. Hit Percent for mean function

\begin{tabular}{|r|c|c|c|c|}
\hline \multicolumn{1}{|c|}{ Filter } & $\begin{array}{c}15^{\circ} \text { change } \\
\text { in viewpoint }\end{array}$ & $\begin{array}{c}34^{\circ} \text { change } \\
\text { in viewpoint }\end{array}$ & $\begin{array}{c}\text { Light from } \\
\text { right } \\
\text { direction }\end{array}$ & $\begin{array}{c}\text { Different front } \\
\text { light source }\end{array}$ \\
\hline Mean Filter & 96.15 & 50 & 0 & 3.84 \\
Histogram Equalized & 84.61 & 46.15 & 0 & 12.23 \\
Giassoam $\sigma=2$ & 84.61 & 50 & 0 & 11.54 \\
Guassian $\sigma=4$ & 84.61 & 50 & 0 & 7.69 \\
\hline
\end{tabular}

\section{Conclusion}

In this work, there is an empirical study on possibility of various image illustrations and checking whether they can be used for palm biometrics is presented. Here, we try to solve some of the challenges faced in build up the biometric applications for civilian use, for forensic use and etc. Recognition of palm print is very crucial work by the hardware. The suggested work identified correct recognition rate and Correction Error rate after extraction of palm images using 2D integration function recognition. Fourier and integration function based palm print features are very important for the image recognition and examination. They play different and equivalent roles in a biometric system. 2D Integration function, the local phase data obtained by the proposed technique is counted as the local feature.

\section{REFERENCES}

[1]. A.-M. Cretu and P. Payeur, Visual attention model with adaptive weighting of conspicuity maps for building detection in satellite image, International Journal on Smart Sensing and Intelligent.

[2]. B. Aleksandra, "Biometric Authentication. Types of biometric identifiers," Helsingfors, 2012.

[3]. W. Kong and D. Zhang, "Palm print texture analysis based on low-resolution images for personal authentication," in Pattern Recognition, 2002. Proceedings. 16th International Conference, Quebec City, Canada, 2002. 
[4]. D. Zhang, W. Kong, J. You and M. Wong, "Online palm print identification," Pattern Analysis and Machine Intelligence, IEEE Transactions, vol. 25, no. 9, pp. 1041-1050, September 2003.

[5]. L. Zhang and D. Zhang, "Characterization of palm prints by wavelet signatures via directional context modeling," IEEE Transactions on Systems Man and Cybrentics - part B: Cybrentics, vol. 34, no. 3, pp. 1335 - 1347, June 2004.

[6]. X. Jing and D. Zhang, "A face and palmprint recognition approach based on discriminant DCT feature extraction," IEEE Trans Syst Man Cybern B Cybern, vol. 34, no. 6, pp. 2405-15, Dec. 2004.

[7]. Vikash Yadav et al, "Detection of Melanoma Skin Disease by Extracting High Level Features for Skin Lesions", International Journal of Advanced Intelligence Paradigms (IJAIP), Vol. 11, No. 3/4, pp. 397-408, September 2018.

[8]. Vikash Yadav et al, "A Hybrid Image Compression Technique for Medical Images", 7th IEEE International Conference on Computational Intelligence and Communication Networks (CICN-2015), pp. 222-227, Organized by Machine Intelligence Research Labs, Jabalpur, India on December 12-14, 2015.

[9]. Vikash Yadav et al, "A Biometric approach to Secure Big Data", International Conference on Innovation and Challenges in Cyber Security (ICICCS-2016), pp. 75-79, Organized by Department of Computer Science \& Engineering, Amity University, Greater Noida, India on February 03-05, 2016.

\section{Cite this article as :}

Abhilove Kumar, Apoorv Mishra, "Palm print Recognition using 2D Fourier Transformation and Integration Function", International Journal of Scientific Research in Computer Science, Engineering and Information Technology (IJSRCSEIT), ISSN : 2456-3307, Volume 7 Issue 3, pp. 555-560, May-June 2021. Available at doi : https://doi.org/10.32628/CSEIT2173165 Journal URL : https://ijsrcseit.com/CSEIT2173165 